\title{
Effect of collisions on amplification of laser beams by Brillouin scattering in plasmas
}

\author{
K. A. Humphrey, ${ }^{1}$ R. M. G. M. Trines, ${ }^{2}$ F. Fiuza,${ }^{3}$ D. C. Speirs,${ }^{1}$ P. Norreys,${ }^{2}$ R. A. Cairns,${ }^{4}$ \\ L. O. Silva, ${ }^{5}$ and R. Bingham ${ }^{1,2}$ \\ ${ }^{1}$ SUPA, Department of Physics, University of Strathclyde, Glasgow G4 ONG, United Kingdom \\ ${ }_{2}^{2}$ STFC Rutherford Appleton Laboratory, Chilton, Didcot, Oxfordshire OX11 0QX, United Kingdom \\ ${ }^{3}$ Lawrence Livermore National Laboratory, Livermore, California 94551, USA \\ ${ }^{4}$ School of Mathematics and Statistics, University of St. Andrews, St. Andrews, Fife KY16 9SS, United Kingdom \\ ${ }^{5}$ GoLP/Centro de Física dos Plasmas, Instituto Superior Técnico, 1049-001 Lisboa, Portugal
}

(Received 7 August 2013; accepted 3 October 2013; published online 17 October 2013)

\begin{abstract}
We report on particle in cell simulations of energy transfer between a laser pump beam and a counter-propagating seed beam using the Brillouin scattering process in uniform plasma including collisions. The results presented show that the ion acoustic waves excited through naturally occurring Brillouin scattering of the pump field are preferentially damped without affecting the driven Brillouin scattering process resulting from the beating of the pump and seed fields together. We find that collisions, including the effects of Landau damping, allow for a more efficient transfer of energy between the laser beams, and a significant reduction in the amount of seed pre-pulse produced. (C) 2013 AIP Publishing LLC. [http://dx.doi.org/10.1063/1.4825356]
\end{abstract}

\section{INTRODUCTION}

The study of parametric instabilities with relevance to interacting laser beams in plasma is of interest to many applications. These include: using Brillouin scattering in plasmas as an amplification technique to generate short and intense light pulses, the study and understanding of complex laser plasma interactions, and induced power transfer between adjacent laser beams as a means of controlling the symmetry of fuel capsule implosion for inertial confinement fusion experiments. ${ }^{1,2}$ Interest in this area has grown due to the potential of similar amplification techniques such as the production of picosecond, kilojoule, and petawatt pulses via Raman scattering ${ }^{3-6}$ and the interaction of multiple laser beams, leading to cross beam energy transfer (CBET), in inertial confinement fusion experiments on the NIF. ${ }^{7,8}$ Following this work, many related numerical or theoretical $^{9-15}$ and experimental ${ }^{16-19}$ studies followed. In particular, a study of laser amplification via stimulated Brillouin scattering (SBS) was conducted by Andreev et al. ${ }^{20}$ however, this work ignored the effects of collisions. Due to the relatively high densities required, typically above one quarter of the critical density, to prevent other processes such as Raman scattering dominating the Brillouin scattering mechanism it is anticipated that collisions will play a significant role in this energy transfer process.

Stimulated Brillouin scattering in plasma, with characteristic electron plasma frequency $\omega_{\text {pe }}$, takes the form of a three-wave interaction where the energy and momentum equations satisfy the Manley-Rowe relations: $\omega_{0}=\omega_{1}+\omega_{\text {IA }}$ and $\mathbf{k}_{0}=\mathbf{k}_{1}+\mathbf{k}_{\mathrm{IA}}$, respectively. Brillouin scattering as an energy transfer and amplification mechanism in plasma uses counter-propagating laser beams where a high frequency laser pump wave, with frequency $\omega_{0}$ and wavenumber $\mathbf{k}_{0}$, is scattered by a low frequency ion-acoustic wave propagating in the same direction as the pump beam, with wavenumber $\mathbf{k}_{\mathrm{IA}} \approx 2 \mathbf{k}_{0}$ and frequency $\omega_{\mathrm{IA}}$ that satisfy the dispersion relation $\omega_{\text {IA }} \approx 2 \mathrm{k}_{0} \mathrm{c}_{\mathrm{s}}$ where $\mathrm{c}_{\mathrm{s}}$ is the ion sound speed, into a laser seed wave, with frequency $\omega_{1}$ and wavenumber $\mathbf{k}_{1}$. As the frequency of the ion acoustic wave is typically much lower than the laser frequency, a large fraction of the energy can be transferred between the laser beams in Brillouin scattering potentially providing a more efficient alternative to Raman amplification.

In this letter, we show that the normal three-wave stimulated Brillouin scattering occurring from the pump field, which prematurely depletes the pump laser before interaction with the seed, can be preferentially damped by the introduction of collisional effects in the code without affecting the driven process, which occurs as a result of the beating of the pump and seed frequencies together. As a result, the energy available for transfer between the beams is maximised and a notable reduction in the amount of seed pre-pulse produced is observed. We also report on the fact that when such collisional effects are accounted for an increase in the plasma ion temperature leads to damping of both of these Brillouin scattering processes resulting in complete eradication of the Brillouin instability. The first results from OSIRIS simulations of the effects of collisional processes and the effect of varying the plasma electron/ion temperature ratio on this energy transfer method through the Brillouin process are presented.

\section{SIMULATIONS}

The energy transfer between two counter-propagating laser pulses was simulated numerically in $1 \mathrm{D}$ using the fully relativistic OSIRIS particle-in-cell code $^{21}$ and was constructed as follows. A pump laser of intensity $10^{16} \mathrm{~W} \mathrm{~cm}^{-2}$ corresponding to a laser wavelength of $1 \mu \mathrm{m}$ was injected in to a plasma column of length $650 \mathrm{c} / \omega_{0}$ with the realistic mass ratio for ions to electrons of $\mathrm{m}_{\mathrm{i}} / \mathrm{m}_{\mathrm{e}}=1836$ used. The plasma temperature ratio of $\mathrm{ZT}_{\mathrm{e}} / \mathrm{T}_{\mathrm{i}}=50$, where $\mathrm{Z}=1$ and $\mathrm{T}_{\mathrm{e}}=500 \mathrm{eV}$ were chosen in order to render Landau damping 
of the ion acoustic wave (IAW) negligible. In all cases, the density was chosen slightly above the quarter critical level at $0.3 n_{c}$ in order to mitigate mode competition from Raman scattering which occurs when the density is $0.25 \mathrm{n}_{\mathrm{c}}$ or below. The seed pulse, of intensity $10^{15} \mathrm{~W} \mathrm{~cm}^{-2}$ and a full width at half maximum (FWHM) of 100 ps with a $\sin ^{2}$ shape, was launched at the instant the pump pulse had traversed the length of the plasma. A frequency mismatch between the pump and seed laser pulses was also introduced, with the seed pulse frequency being downshifted by an amount equal to the ion acoustic frequency. The pulses are counter propagating through the plasma with the pump pulse travelling from right to left through the simulation box. The time step for integration is $\Delta t=0.04 \omega_{0}{ }^{-1}$ and the spatial resolution of the simulations is of the order of the Debye length with 100 particles per cell. In the simulations where collisional processes were included, these were calculated self-consistently by OSIRIS for a reference plasma density of $3 \times 10^{20} \mathrm{~cm}^{-3}$ corresponding to a group speed of approximately $0.84 c$ for both pulses.

\section{COLLISIONLESS CASE}

Figure 1 shows the evolution of the transverse electric field as the seed and pump fields interact via stimulated Brillouin scattering for the case where collisional effects are neglected. Energy transfer from the pump to the seed is observed, whilst the seed duration is approximately constant avoiding amplification via compression of the pulse. After approximately $1840 \omega_{\mathrm{p}}{ }^{-1}$, the seed pulse reaches maximum amplitude, after which the energy transfer process begins to degrade and the pulse loses its integrity. It can clearly be seen that the pump field is significantly depleted behind the leading edge of the seed indicating a that a significant fraction of the available energy in the pump field has been transferred to the seed. It is also noted, however, that there is a large proportion of seed pre-pulse generated when collisional effects are neglected which is particularly unfavorable for use in laser-plasma applications. The formation of this pre-pulse is as a direct result of the pump/seed interaction as no pre-pulse formation is observed when a single laser pulse is utilized.

\section{COLLISIONAL CASE}

Analysis of the phase space plots for this process strongly indicates the excitation and growth of a number of IAW's associated with the Brillouin scattering mode. Figure 2 shows phase space plots for the plasma ion population from the point where the energy transfer process is initiated up until the seed has reached maximum intensity. On the left hand side of each of the plots in Figure 2, the IAW corresponding to the driven process, resulting from the beating of the seed and pump fields together, is seen to be excited and grow steadily until a time of $\mathrm{t}=1840 \mathrm{\omega}_{\mathrm{p}}{ }^{-1}$, commensurate with the saturation of the energy transfer between the seed and pump. It can also be seen, however, that in addition to the excitation of the beat ion acoustic wave associated with the driven process a number of other, naturally occurring, IAW's are also excited. These waves can be seen to emanate from the RHS of the simulation volume and are due to the pump laser undergoing naturally occurring stimulated Brillouin scattering before the pump/seed pulse interaction. Without collisional damping, the threshold for stimulated Brillouin scattering is effectively zero which results in growth of ion acoustic waves and subsequent depletion of the pump wave before the pump and the seed overlap as the pump drives up ion-acoustic waves and generates a backward propagating scattered wave. This has an adverse effect on the pump/seed energy transfer efficiency.

Analysis of the effect of including collisional processes into the simulation setup can be seen in Figure 3. Upon comparison of these transverse electric field plots to the plots at
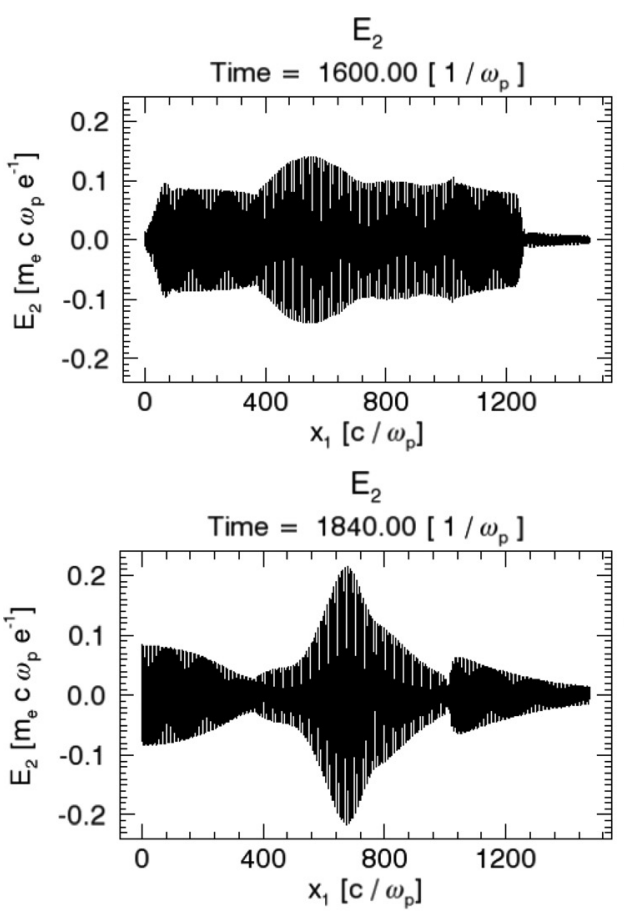

$E_{2}$

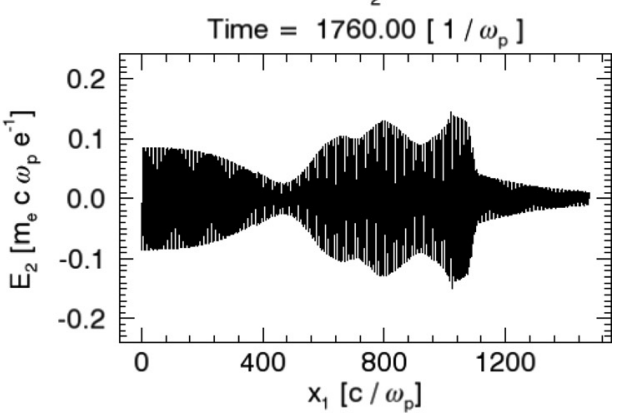

$\mathrm{E}_{2}$

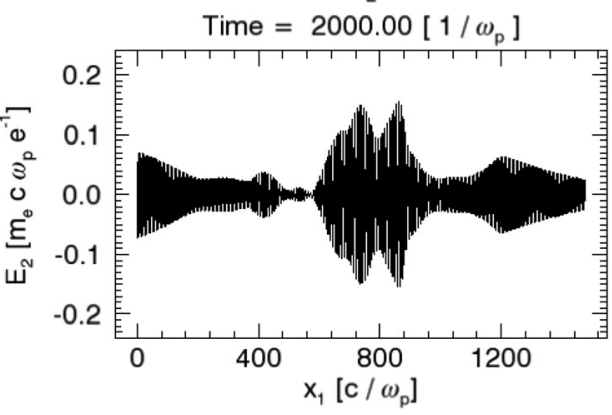

FIG. 1. Transverse electric field profile at $\mathrm{t}=1600,1760,1840$, and 2000 $\omega_{\mathrm{p}}{ }^{-1}$ for PiC simulation with collisional effects neglected. 

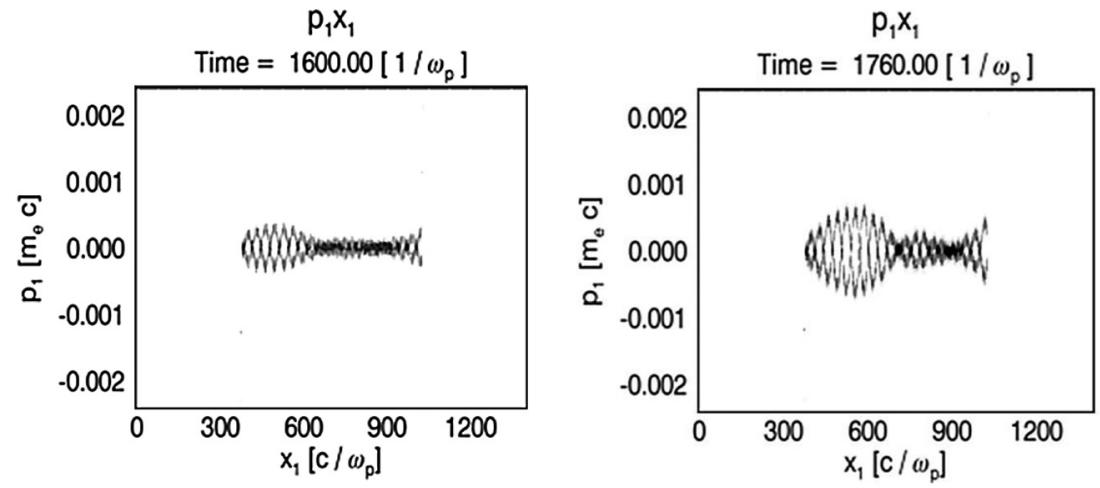

$p_{1} x_{1}$

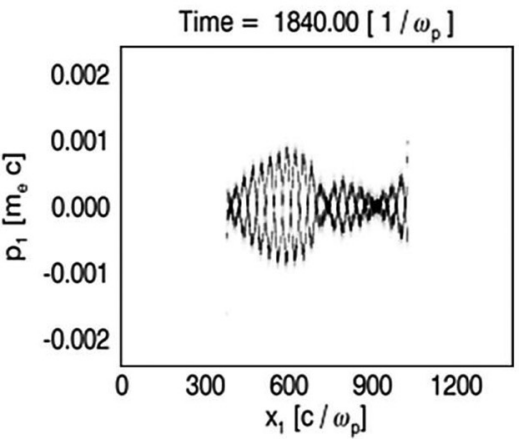

$p_{1} x_{1}$

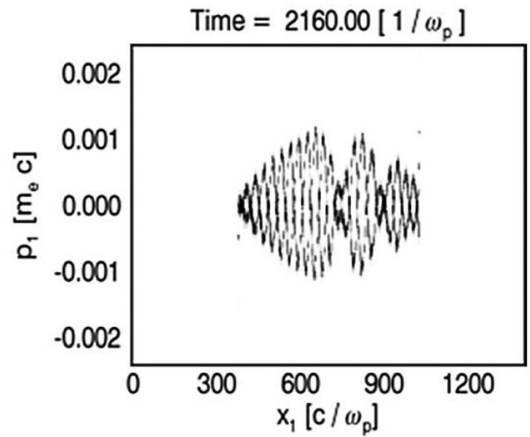

FIG. 2. Ion phase space plots at $\mathrm{t}=1600,1760,1840$, and $2160 \omega_{\mathrm{p}}{ }^{-1}$ for $\mathrm{PiC}$ simulation with collisional effects neglected. the same timestep in Figure 1, associated with the collisionless setup, it can be seen that there are some significant differences. It can be seen that the growth of the energy transfer process is slower; meaning the seed pulse takes longer to reach maximum amplitude. Due to this reduced growth, the resultant seed has a longer duration as it takes longer to deplete the pump field. As a result of this, the pulse has split into a number of beamlets, resulting in a marginal decrease in the final seed amplitude from that of the collisionless case. The efficiency of the process is found to be $10 \%$ higher and this is attributed to the fact that the Brillouin scattering process resulting from the pump field is no longer able to prematurely deplete the energy available for transfer to the seed pulse causing degradation in the energy transfer process. Again, it is seen that the portion of the pump laser field which has interacted with the seed pulse is significantly depleted indicating that the energy transfer mechanism remains efficient with the thermal effects included in the code. Of particular interest is the significant reduction in the volume of seed pre-pulse generated from stimulated Brillouin scattering when the collisional processes are accounted for. The introduction of collisions therefore provides a significant improvement in the contrast of the laser beam with a very small sacrifice in the resultant laser

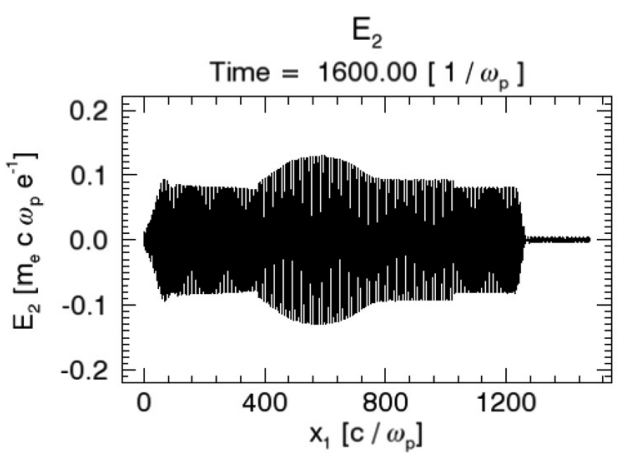

$\mathrm{E}_{2}$

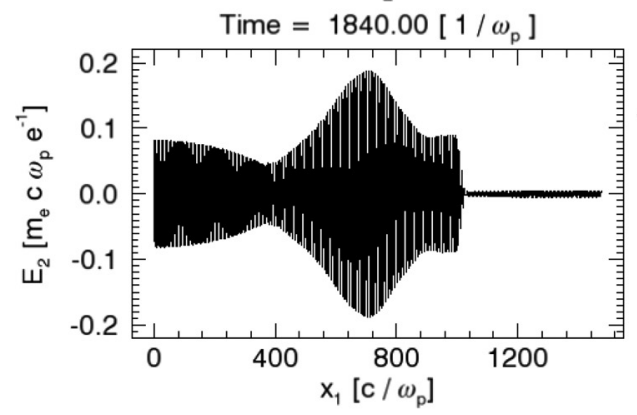

$\mathrm{E}_{2}$

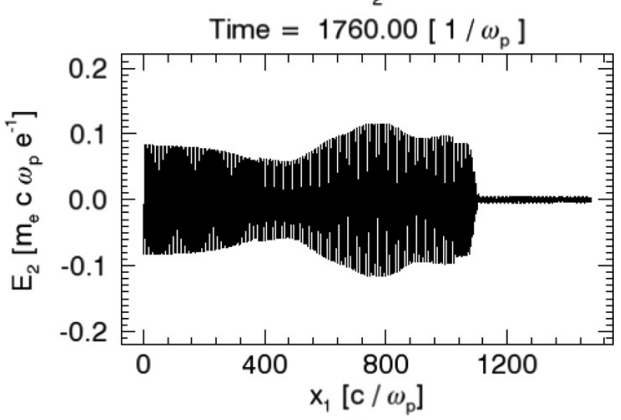

$\mathrm{E}_{2}$

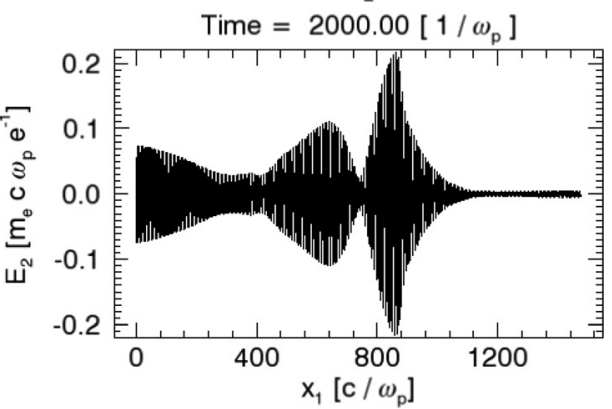

FIG. 3. Transverse electric field profile at $\mathrm{t}=1600,1760$, and 1840 and 2000 $\omega_{\mathrm{p}}{ }^{-1}$ for $\mathrm{PiC}$ simulation with c ollisional effects included. 

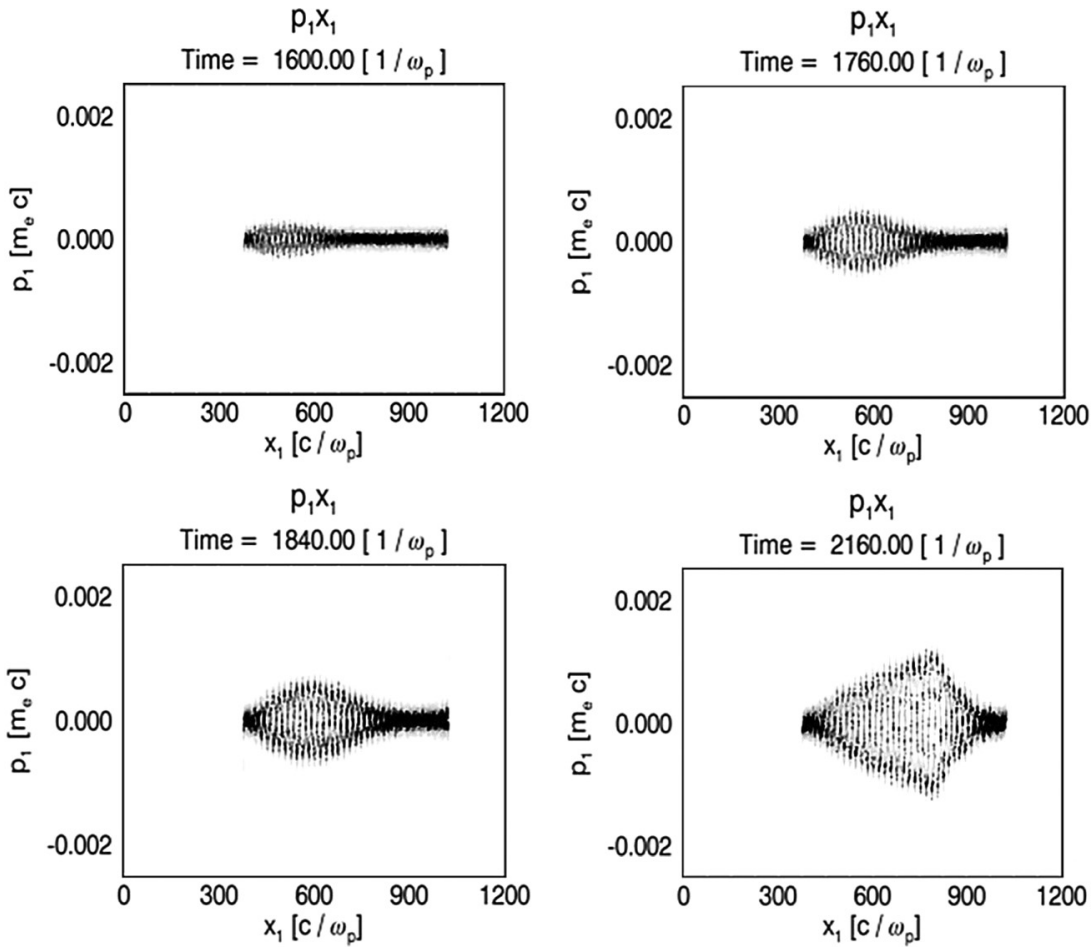

FIG. 4. Ion phase space plots at $\mathrm{t}=1600,1760,1840$, and $2160 \omega_{\mathrm{p}}{ }^{-1}$ for $\mathrm{PiC}$ simulation with collisional effects included. amplitude, compared with the same pump length, for amplified pulses resulting from stimulated Brillouin scattering.

The most significant difference between the two cases can be observed from Figure 4, when compared directly with the plots at the same time iterations in Figure 2, where it can be seen that the naturally growing IAW's previously seen on the RHS of the simulation box have vanished. This is due to the fact that by including collisional processes into the simulation we encounter a threshold for the normal three-wave scattering process that prevents mode competition between the beat-wave driven and naturally occurring Brillouin scattering mechanisms. In the absence of collisions, there is no such threshold meaning that both the naturally occurring three-wave Brillouin scattering and the beat wave Brillouin scattering process are present. By damping the unwanted normal three-wave Brillouin process, resulting from the scattering of the pump field before the pump/seed laser interaction, premature depletion of the pump laser can be minimized ensuring that the energy available for exchange between the pump and seed lasers is maximized. In addition to the difference in growth rates of the IAW's generated from both the collisionless and collisional simulations, an analysis of the individual IAW oscillations generated from both studies of Brillouin scattering show that in both cases particles are accelerated, indicating the initial signs of wave breaking causing the onset of the saturation of the energy transfer mechanism. ${ }^{20}$

An investigation was also conducted to assess the effect of various $T_{e} / T_{i}$ temperature ratios on the beat wave driven Brillouin scattering process. It was found that as the plasma ion temperature was increased such that it approached the temperature of the plasma electron species, it was found that the IAW's waves were increasingly Landau damped such that both the beat wave driven and naturally occurring Brillouin scattering processes were suppressed. This is particularly interesting result as it is seen that for high enough plasma ion temperatures that the Brillouin instability can be completely damped and unwanted energy exchange between beams can be avoided for applications where SBS is undesirable.

\section{SUMMARY}

In summary, we have investigated the effect of collisional processes on the energy transfer between laser pulses by stimulated Brillouin scattering. We have shown that, for a constant pump to probe ratio, collisional effects are found to damp the normal three-wave Brillouin scattering process ensuring that the pump laser is unable to scatter before interaction with the seed pulse. In addition to this, we have observed the introduction of collisions results in an increase in the efficiency of stimulated Brillouin scattering and a significant increase in the contrast of the resultant seed laser beam. We have also discussed the effect of raising the plasma ion temperature, such that the ion acoustic waves associated with both the normal three wave Brillouin scattering and the beat wave driven Brillouin scattering processes are Landau damped mitigating the onset and subsequent growth of the instability. These are significant new results and this work has important consequences for the future of ultra-high intensity laser systems and their applications towards pulse energy transfer and amplification.

\section{ACKNOWLEDGMENTS}

The authors would like to thank the OSIRIS consortium for the use of OSIRIS and the STFC Centre for Fundamental Research for their support. Simulations were carried out on the Scarf-Lexicon Cluster (STFC RAL). Authors KH, RT, DCS, RAC, RB were supported by EPSRC grant EP/G04239X/1. 
${ }^{1}$ J. D. Lindl, P. Amendt, R. L. Berger, S. G. Glendinning, S. H. Glenzer, S. W. Haan, R. L. Kauffman, O. L. Landen, and L. J. Suter, Phys. Plasmas 11, 339 (2004).

${ }^{2}$ S. H. Glenzer, D. H. Froula, L. Divol, M. Dorr, R. L. Berger, S. Dixit, B. A. Hammel, C. Haynam, J. A. Hittinger, J. P. Holder, O. S. Jones, D. H. Kalantar, O. L. Landen, A. B. Langdon, S. Langer, B. J. MacGowan, A. J. Mackinnon, N. Meezan, E. I. Moses, C. Niemann, C. H. Still, L. J. Suter, R. J. Wallace, E. A. Williams, and B. K. F. Young, Nature Phys. 3, 716 (2007).

${ }^{3}$ R. M. G. M. Trines, F. Fiuza, R. Bingham, R. A. Fonseca, L. O. Silva, R. A. Cairns, and P. A. Norreys, Phys. Rev. Lett. 107, 105002 (2011).

${ }^{4}$ R. M. G. M. Trines, F. Fiuza, R. Bingham, R. A. Fonseca, L. O. Silva, R. A. Cairns, and P. A. Norreys, Nature Phys. 7, 87 (2011).

${ }^{5}$ G. Shvets, N. J. Fisch, A. Pukhov, and J. Meyer-ter-Vehn, Phys. Rev. Lett. 81, 4879 (1998).

${ }^{6}$ V. M. Malkin, G. Shvets, and N. J. Fisch, Phys. Rev. Lett. 82, 4448 (1999).

${ }^{7}$ L. Kruer, S. C. Wilks, B. B. Afeyan, and R. K. Kirkwood, Phys. Plasmas 3, 382 (1996).

${ }^{8}$ R. K. Kirkwood, B. B. Afeyan, W. L. Kruer, B. J. MacGowan, J. D. Moody, D. S. Montgomery, D. M. Pennington, T. L. Weiland, and S. C. Wilks, Phys. Rev. Lett. 76, 2065 (1996).

${ }^{9}$ D. M. Villeneuve, H. A. Baldis, and J. E. Bernard, Phys. Rev. Lett. 59, 1585 (1987).

${ }^{10}$ V. V. Eliseev, W. Rozmus, V. T. Tikhonchuk, and C. E. Capjack, Phys. Plasmas 3, 2215 (1996).

${ }^{11}$ C. J. McKinstrie, J. S. Li, R. E. Giacone, and H. X. Vu, Phys. Plasmas 3, 2686 (1996).

${ }^{12}$ P. Michel, L. Divol, E. A. Williams, S. Weber, C. A. Thomas, D. A. Callahan, S. W. Haan, J. D. Salmonson, S. Dixit, D. E. Hinkel, M. J.
Edwards, B. J. MacGowan, J. D. Lindl, S. H. Glenzer, and L. J. Suter, Phys. Rev. Lett. 102, 025004 (2009).

${ }^{13}$ P. Michel, S. H. Glenzer, L. Divol, D. K. Bradley, D. Callahan, S. Dixit, S. Glenn, D. Hinkel, R. K. Kirkwood, J. L. Kline, W. L. Kruer, G. A. Kyrala, S. Le Pape, N. B. Meezan, R. Town, K. Widmann, E. A. Williams, B. J. MacGowan, J. Lindl, and L. J. Suter, Phys. Plasmas 17, 056305 (2010).

${ }^{14}$ B. I. Cohen, B. F. Lasinski, A. B. Langdon, E. A. Williams, K. B. Wharton, R. K. Kirkwood, and K. G. Estabrook, Phys. Plasmas 5, 3408 (1998).

${ }^{15}$ E. A. Williams, B. I. Cohen, L. Divol, M. R. Dorr, J. A. Hittinger, D. E. Hinkel, A. B. Langdon, R. K. Kirkwood, D. H. Froula, and S. H. Glenzer, Phys. Plasmas 11, 231 (2004).

${ }^{16}$ K. B. Wharton, R. K. Kirkwood, S. H. Glenzer, K. G. Estabrook, B. B. Afeyan, B. I. Cohen, J. D. Moody, and C. Joshi, Phys. Rev. Lett. 81, 2248 (1998).

${ }^{17}$ R. K. Kirkwood, J. D. Moody, A. B. Langdon, B. I. Cohen, E. A. Williams, M. R. Dorr, J. A. Hittinger, R. Berger, P. E. Young, L. J. Suter, L. Divol, S. H. Glenzer, O. L. Landen, and W. Seka, Phys. Rev. Lett. 89, 215003 (2002).

${ }^{18}$ C. Labaune, H. A. Baldis, E. Schifano, B. S. Bauer, A. Maximov, I. Ourdev, W. Rozmus, and D. Pesme, Phys. Rev. Lett. 85, 1658 (2000).

${ }^{19}$ L. Lancia, J. R. Marques, M. Nakatsutsumi, C. Riconda, S. Weber, S. Huller, A. Mancic, P. Antici, V. T. Tikhonchuk, A. Heron, P. Audebert, and J. Fuchs, Phys. Rev. Lett. 104, 025001 (2010).

${ }^{20}$ A. Andreev, C. Riconda, V. T. Tikhonchuk, and S. Weber, Phys. Plasmas 13, 053110 (2006).

${ }^{21}$ R. A. Fonseca, L. O. Silva, F. S. Tsung, V. K. Decyk, W. Lu, C. Ren, W. B. Mori, S. Deng, S. Lee, T. Katsouleas, and J. C. Adam, in Computational Science-ICCS 2002, Lecture Notes in Computer Science, Vol. 2331, edited by P. M. A. Sloot, C. J. K. Tan, J. J. Dongarra, and A. G. Hoekstra (Springer, 2002), pp. 342-351. 